\title{
Rotator Cuff Repair with Different Suture Materials Results in Different Protein Expressions with Similar Histological Findings: An Experimental Study in Rabbits
}

\author{
E. Okay ${ }^{1}$, M. Bezer ${ }^{2}$ \\ 1 Department of Orthopaedics, Istanbul Medeniyet University Goztepe Education and Research Hospital, \\ Istanbul, Turkey \\ 2 Department of Orthopaedics, Fevzi Çakmak Marmara University Education and Training Hospital, Pendik, \\ Istanbul, Turkey
}

\section{CORRESPONDING AUTHOR: \\ Erhan Okey \\ Department of Orthopaedics \\ Istanbul Medeniyet University Goztepe \\ Education and Research Hospital \\ Kadıköy \\ 34722 Istanbul, Turkey \\ E-mail: erhanokay@yahoo.com}

DOI:

10.32098/mltj.04.2020.07

LEVEL OF EVIDENCE: 2B

\section{SUMMARY}

Background. Previous studies have evaluated the biomechanical, histological, and clinical effects of rotator cuff repair with different suture materials. However, a histological and western blot analysis after repair comparing nonabsorbable polyester and polyethylene suture materials in a chronic rotator cuff tear model has not been conducted in any previous studies. The aim of this study is to evaluate the effect the two different suture materials, specifically Ethibond and Ultrabraid, have on repair based on a histological evaluation and protein expression by means of a western blot analysis.

Methods. Twenty-six rabbits were included in the study. Two rabbits served as normal controls (control group). A bilateral tenotomy of the subscapularis tendon was performed in the remaining 24 rabbits. Six weeks after the tenotomy, the animals were randomly divided into four repair groups (five rabbits in each group) according to the suture material used and the sacrifice time (Ultrabraid two weeks after repair, Ethibond two weeks after repair, Ultrabraid six weeks after repair, and Ethibond six weeks after repair). Two sham-operated groups with two rabbits each were sacrificed at eight and 12 weeks after the tenotomy without repair. Both shoulders of all the animals were used for a western blot (collagen type 1-3, MMP-2, TGF-beta-1, IGF-1, COX-2) analysis and a histological evaluation (fibrocartilage formation, inflammation, vascularisation, collagen density).

Results. All groups showed minimal-to-mild histological changes irrespective of the type of suture material used. There was no statistically significant difference in histological scores $(p>0.05)$. Fibrocartilage formation was greater at six weeks in the polyester suture repair groups without statistical significance $(\mathrm{p}>0.05)$. Collagen type 1-3, MMP-2, TGF-beta-1, IGF-1, and COX-2 were significantly upregulated at two weeks compared to six weeks after repair $(\mathrm{p}<0.05)$. Protein expression levels were statistically significantly higher in the Ultrabraid groups compared to the Ethibond groups $(\mathrm{p}<0.05)$.

Conclusions. Changes in the expression of the markers related to rotator cuff healing were demonstrated when repairing with different suture materials, with these being in favour of polyester sutures; however, the histological findings are similar. This finding could influence the design of new suture materials.

\section{KEY WORDS}

Fibrocartilage formation; rabbit rotator cuff bone-tendon healing; suture material; western blot analysis; MMP-2; COX-2. 


\section{INTRODUCTION}

The non-healing rate after rotator cuff repair has been reported as being between $20 \%$ and $70 \%(1,2)$. Several factors, such as increased age, chronic diseases, quality of tendon, smoking, and obesity, have been demonstrated to be related to a failure to heal after rotator cuff repair (3-6). The use of different suture materials has also been thought to influence healing (7). In regard to nonabsorbable sutures, polyblend (Fiberwire, Herculine, Orthocord, and Ultrabraid) and conventional polyester (Ethibond) suture materials are used in rotator cuff surgery (8). In the last decade, polyblend sutures comprising Ultra-High-Molecular-Weight Polyethylene (UHMWPE) have become preferred because of their enhanced biomechanical properties (9). Polyblend sutures' biomechanical superiority to polyester suture materials has been previously demonstrated (10).

A recent in vitro study by Lock et al. showed changes in the synthesis of inflammatory markers at one, three, and five days after suture placement (11). In an acute tendon rupture model, Ergun et al. performed Achilles tendon repair with different suture materials. It was found that the biological properties of absorbable PDS sutures were superior compared to Vicrl and Ethibond sutures, and the study suggests using absorbable PDS sutures in Achilles tendon repairs (12). Su et al. investigated the effect of suture absorbability on enthesis regeneration and biomechanical properties in an acute rotator cuff healing model in rabbits (7). Repair with absorbable sutures rather than partially absorbable (Orthocord) or nonabsorbable (Ethibond) sutures was shown to evoke enthesis regeneration. Due to their possible stress-shielding effect, the healing properties of nonabsorbable sutures have been found to be inferior to those of absorbable and partially absorbable sutures. However, this study was performed on an acute rotator cuff tear model.

To date, biomechanical comparisons of suture cut-through (13), the influence of knot location (14), and the suture absorbability (7) of different suture materials in acute rotator cuff healing have been studied; however, whether the type of suture material used in surgery would affect chronic rotator cuff healing on histological and molecular levels remains unknown.

Therefore, this study aims to compare the healing effect of repair with two different suture materials, specifically Ethibond and Ultrabraid, at different intervals (at two and six weeks after repair) using histologic and western blot analysis. It was hypothesised that transosseous repair with different suture materials would influence the histologic findings and protein expressions, which are thought to have a role in catabolic, anabolic, and remodelling processes, during rotator cuff healing.

\section{METHODS}

This is a committee-approved study using an established rabbit rotator cuff repair model. A rabbit subscapularis chronic tear model was chosen because of comparable histologic and biomechanical similarities to human chronic rotator cuff pathology (15-18). All institutional and national guidelines for the care and use of laboratory animals were followed. Approval for this study was obtained from the Ethics Committee of Pendik Veterinary Control and Research Institute, Istanbul, Turkey. A priori power analysis indicated that a total of 26 animals were required. Twenty-six mature New Zealand White male rabbits with an average age of 36 weeks and a weight of $2.5 \mathrm{~kg}$ to $3.5 \mathrm{~kg}$ were included in the study.

\section{Study design}

Twenty-six rabbits were included in the study. Two rabbits in the control group did not undergo surgery. A bilateral tenotomy of the subscapularis tendon was performed in the remaining 24 rabbits. Six weeks after the tenotomy, secondary repair procedures were performed bilaterally. The rabbits were randomly divided into four groups of five rabbits each according to the suture type and euthanasia time as follows: Ethibond repair group (at two weeks after repair), Ethibond repair group (at six weeks after repair), Ultrabraid repair group (at two weeks after repair), and Ultrabraid repair group (at six weeks after repair). Six weeks after the tenotomy, the repair was made using either pure braided UHMWPE polyethylene (Ultrabraid) or braided polyester suture (Ethibond) in suture repair groups with five rabbits each. Two groups with two rabbits each were allocated as sham-operated groups without repair according to the after-sacrifice time, which was at eight weeks and 12 weeks after the tenotomy. A western blot analysis (collagen type I-III, Matrix Metalloproteinase-2 (MMP-2), Transforming Growth Factor (TGF)-beta-1, Insulin-like Growth Factor (IGF)-1, and Cyclooxygenase (COX)-2) and a histologic evaluation (inflammation, vascularisation, and collagen density) were conducted for all groups (16). For the repair groups, the fibrocartilage formation was evaluated using a quantitative histologic analysis.

\section{Surgical technique}

All of the surgical procedures were performed by the same surgeon. Anaesthesia was induced using an intramuscular injection of $35 \mathrm{mg} / \mathrm{kg}$ ketamine and $5 \mathrm{mg} / \mathrm{kg}$ xylazine hydrochloride. After skin preparation, an anterior transverse incision was made at the glenohumeral joint (figure $1 \mathrm{~A}$ ). The deltopectoral interval and the coracobrachialis muscle were 


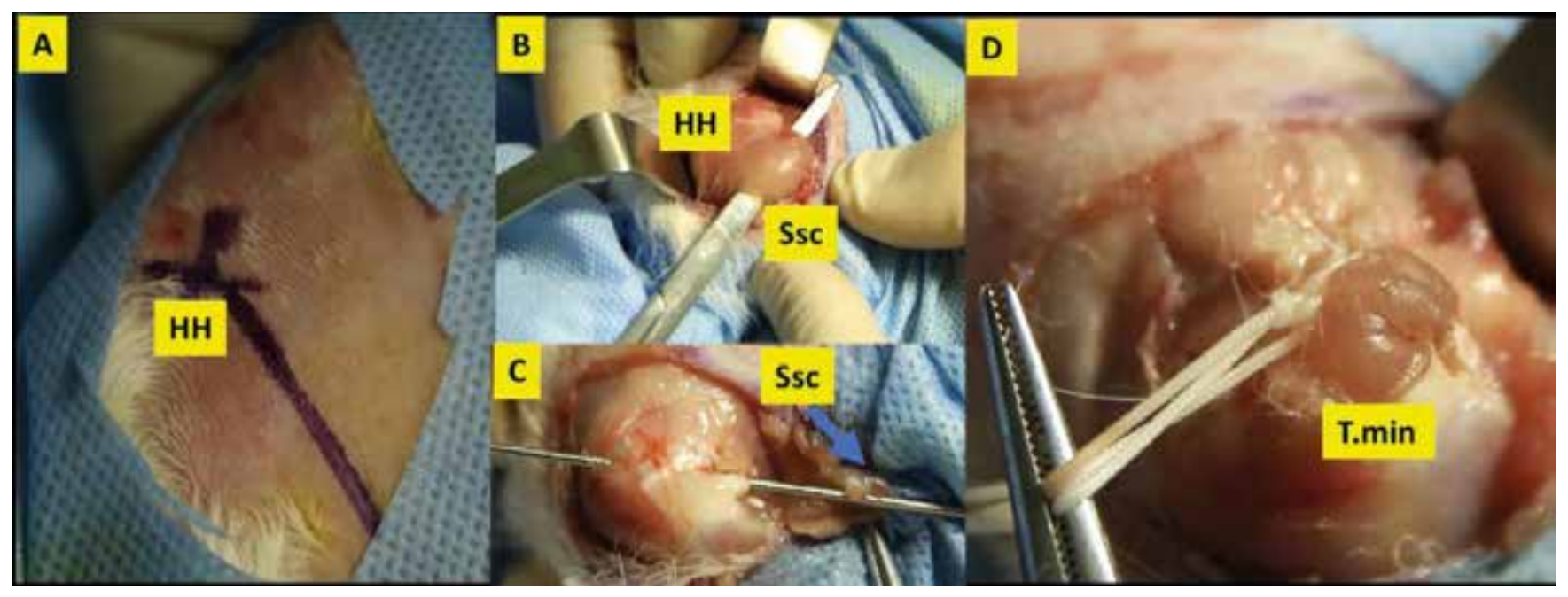

Figure 1. (A) Surgical planning, (B) identification of subscapularis tendon, (C) preparation of bony tunnel using 0.8 mm Kirschner wire, and (D) transosseous repair of the subscapularis tendon. Blue arrow indicates the subscapularis muscle. $\mathrm{HH}$ : Humeral Head; Ssc: Subscapularis muscle; T.min: Tuberculum minus.

split. The subscapularis muscle under the spina scapula was identified using a right angle clamp (figure 1 B). The insertion of the subscapularis at the lesser tubercle of the humerus was sharply released with a scalpel. Six weeks after the tenotomy, the repair was performed using simple transosseous stitches in a Mason-Allen configuration and either No. 2 Ethibond Excel (Ethicon, Somerville, NJ) or No. 2 Ultrabraid Cobraid (Smith \& Nephew, Memphis, Tennessee) sutures were used (figure 1 C-D). The control group was sacrificed without tenotomy or repair to provide a baseline evaluation. The other subjects in the sham-operated and suture repair groups were immobilised after surgery until euthanasia. This was accomplished by positioning a Vetflex elastic bandage (Kruuse group, Langeskov, Denmark) over the forelimb, shoulder, and torso, with both limbs placed at $90^{\circ}$ of forward elevation $20^{\circ}$ of abduction, which has been shown to have superior healing characteristics compared to postoperative mobilisation (19). During the first round of operations, three rabbits died, and one rabbit developed foot drop secondary to iatrogenic injury. Four new rabbits were added to the relevant groups. There was no change in the weight and health status of the entire study group.

\section{Histologic evaluation}

After euthanasia, five bone-tendon specimens from each group underwent histologic analysis. The specimens were fixed in $4 \%$ formic acid-sodium citrate solution for 45 days. The specimens were washed with flowing water for one day. Following dehydration in an alcohol series and clearing with xylene, the samples were embedded in paraffin blocks, which were cut through the longitudinal axis to the subscapularis tendon. Five-micrometre bone-subscapularis tendon sections were taken and placed onto slides. The macroscopic evaluation included the presence of a retear, scar formation, and mode of suture failure, either suture slippage or suture breakage, by observing the suture-tendon interface of each specimen. The sections were stained using Masson's trichrome for collagen fibril density and Safranin-O for inflammation, vascularisation, and collagen fibril density. The histologic criteria reported by Chung et al. were modified and used for the evaluation (20). The histologic examinations evaluated collagen fibre density, presence of inflammatory cells, and vascularity. The scores were graded as absent or minimally present ( 0 points), mildly present (1 point), moderately present ( 2 points), and severe or markedly present (3 points).

Regarding the repair groups, the fibrocartilage formation was evaluated using a previously reported technique (21). The areas of metachromasia were outlined on Safranin-O fast green slides at 100x magnification. The total area of fibrocartilage is provided with percentages as compared to the control groups. All slides were examined by two blinded histologists who were not included in the study. Regions of Interest (ROIs) were imaged at a magnification of 100x. To eliminate observer bias, one histologist performed a general histologic evaluation, and the other histologist performed the quantitative evaluation for fibrocartilage formation. Each slide was coded with randomly generated numbers. Images of three sequential sections were obtained from each 
specimen under the same conditions to reduce sampling error. The results of these sections for each sample were averaged for histologic scoring and quantitative evaluation.

\section{Western blot}

After euthanasia, a western blot analysis of five samples from the musculotendinous junction for each group, including the suture-tendon samples, was performed. One hundred milligram of tissue for each specimen was lysed using 2 $\mathrm{mL}$ lysis buffer. After centrifugation at 15,000 rpm for 20 minutes, the proteins of the lysates were quantified using a BCA Protein Assay Kit (Thermo Fisher Scientific, IL, USA). Equal amounts of total protein $(30 \mu \mathrm{g})$ were run on $10 \%$ SDS polyacrylamide gels at a constant voltage of $200 \mathrm{~V}$ for 40 minutes and transferred onto a nitrocellulose membrane. After blocking the membranes with 5\% milk for $10 \mathrm{~min}$, they were incubated with specific primary antibodies of MMP-2, IGF-1, COX-2, collagen type I alpha 1, collagen type III alpha 1, and TGF-beta 1 at $4{ }^{\circ} \mathrm{C}$ overnight and then washed with Tris-Buffered Saline (TBS) containing 0.1\% Tween-20. After washing with TBS, the membranes were developed using an enhanced chemiluminescent detection kit (Santa Cruz Biotechnology, Texas, USA) against Horseradish Peroxidase (HRP) and then photographed using a ChemiDoc XRS system (Bio-Rad). The signal intensity of the digital blots was measured using processing and analysis software to generate semiquantitative data from the western blot images. An antibody against Glyceraldehyde 3-Phosphate Dehydrogenase (GAPDH) (Novus Biologicals, USA) was used for normalisation of the protein loading on the blots.

\section{Statistics and data analysis}

The data on the histologic parameters and western blot analysis is provided as mean and standard deviations. Group differences were compared using the Kruskal-Wallis test, followed by post-hoc Mann-Whitney U testing with Bonferroni correction. A value of $\mathrm{p}<0.05$ was considered statistically significant. NCSS statistical software (version 2007, NCSS LLC, Kaysville, Utah, USA) was used to perform the analyses.

\section{RESULTS}

\section{Histology}

On macroscopic evaluation, all specimens were intact. Scar tissue was present in suture-tendon areas. None of the samples displayed bone-tendon dehiscence, suture pull- out, or suture breakage. An infected appearance was not observed. There was no gap formation in the bone-tendon areas (figure 2). Safranin-O staining showed the formation of four typical areas, namely tendon, demineralised fibrocartilage, mineralised fibrocartilage, and bone, in the enthesis sites (figure 3). Masson-trichrome staining showed increased collagen fibril density and parallel orientation of collagen over time for each group, with more density in the Ethibond repair groups (figure 4). The histologic healing scores are shown in figure $\mathbf{5}$. For both suture groups, the amount of fibrocartilage at six weeks was more than at two weeks. At each time point, more fibrocartilage had formed in the Ethibond suture groups compared to the Ultrabraid suture groups. The comparison of healing scores and fibrocartilage formation for Ultrabraid versus Ethibond in terms of repair period, i.e. two weeks versus six weeks, was not statistically significant $(p>0.05)$ (figure 6). At each time point, fibrocartilage formation (two weeks: $\mathrm{p}=0,137$, six weeks: $\mathrm{p}=0,111 ; \mathrm{p}>0.05$ ), inflammation (two weeks: $\mathrm{p}=0,118$; six weeks: $\mathrm{p}=0,111 ; \mathrm{p}>0.05$ ), vascularisation (two weeks and six weeks: 0,$546 ; \mathrm{p}>0.05$ ), and collagen density (two weeks: $\mathrm{p}=0,475$, six weeks: $\mathrm{p}=0,319 ; \mathrm{p}>0.05)$ were similar in the Ultrabraid and Ethibond groups ( $\mathrm{p}>0.05$ ) (figure 6).

\section{Protein expression}

A western blot analysis was performed for all groups. At each time point, collagen I expression was higher in the Ethibond groups than in the Ultrabraid groups ( $\mathrm{p}=0.021 ; \mathrm{p}$ $<0.05$ ). At each time point, collagen I expression was higher in the Ethibond groups than in the Ultrabraid groups $(\mathrm{p}=0.020 ; \mathrm{p}<0.05)$. At each time point, IGF-1 (two weeks:

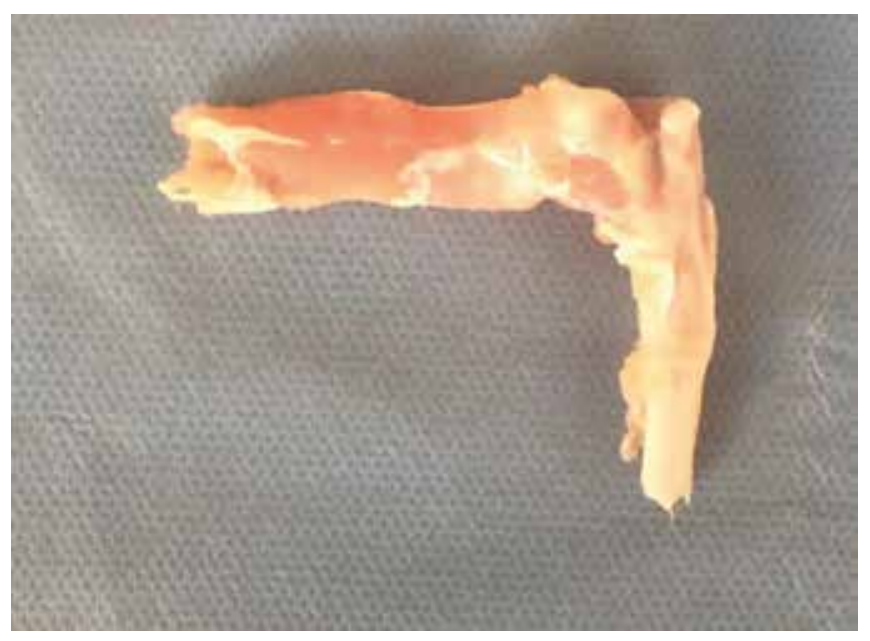

Figure 2. Macroscopic view of the subscapularis tendonbone specimen. 


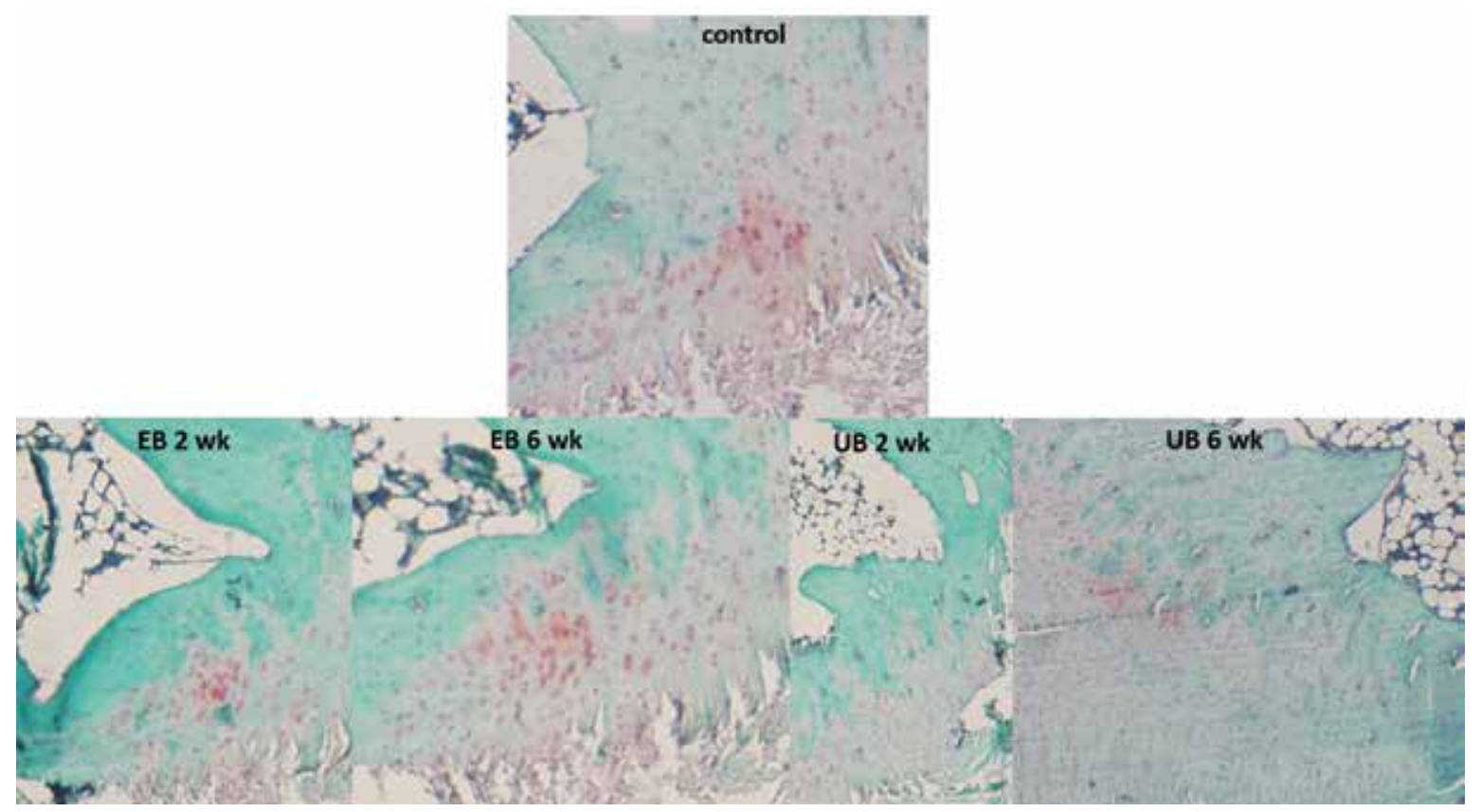

Figure 3. Safranin-O staining of the healing enthesis for the two suture materials at two weeks and six weeks after repair. Area of metachromasia is depicted in red (at an original magnification of 100x). EB: Ethibond; UB: Ultrabraid.

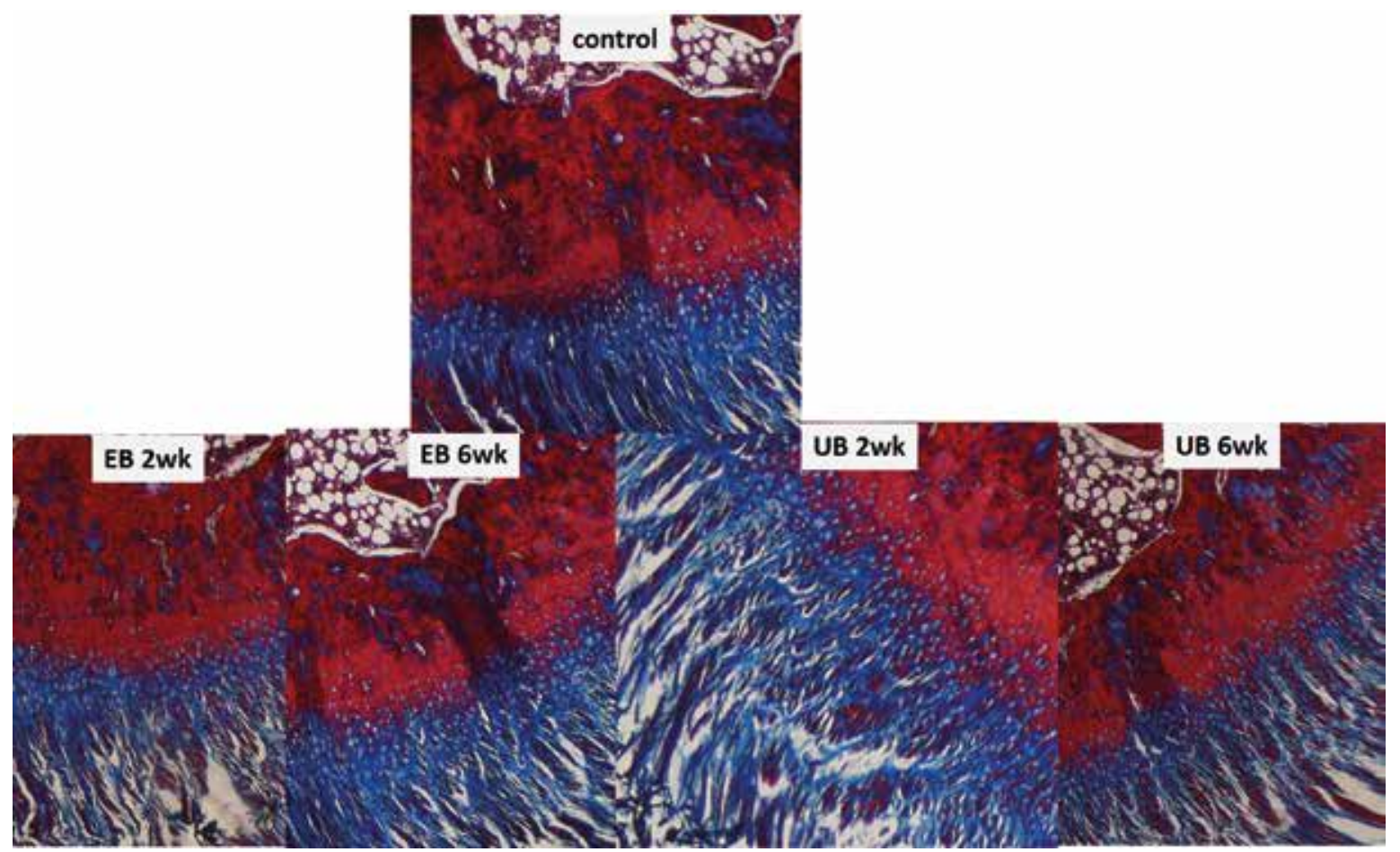

Figure 4. Masson trichrome staining for the evaluation of the collagen density at two weeks and six weeks after repair with the two suture materials (at an original magnification of 100x). At six weeks, more collagen fibres became evident at the tendon area of the newly formed enthesis.

EB: Ethibond; UB: Ultrabraid. 


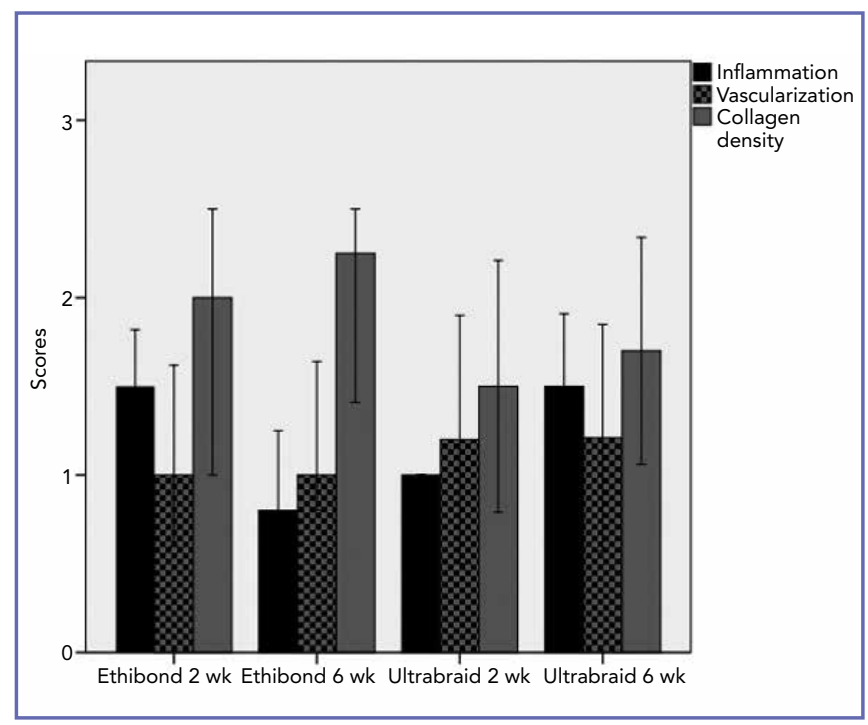

Figure 5. Comparison of the fibrocartilage formation in all the suture groups. Values are given as percentages relative to the control.

$\mathrm{p}=0.043$, six weeks: $\mathrm{p}=0.021 ; \mathrm{p}<0.05$ ), COX-2 (two weeks: $\mathrm{p}=0.021$; six weeks: $\mathrm{p}=0.014 ; \mathrm{p}<0.05$ ), MMP-2 (two weeks and six weeks: $\mathrm{p}<0.001$ ), and TGF-beta 1 (two weeks and six weeks: $\mathrm{p}=0.021 ; \mathrm{p}<0.05)$ expression were all higher in the Ultrabraid groups than in the Ethibond groups $(\mathrm{p}<$ 0.05) (figure 7).

\section{DISCUSSION}

This study shows that repair with Ethibond, a first-generation high-strength polyester suture, and Ultrabraid, a new type of high strength polyblend suture, demonstrates similar histologic changes at six weeks after repair; however, the protein expression levels in relation to rotator cuff healing were altered.

The histologic data is consistent with findings demonstrated by previous animal models of rotator cuff injury $(14,22)$. With various research purposes, several animal models of rotator cuff injury have evaluated healing and control subjects that had undergone rotator cuff repair with different suture materials $(7,14,22)$. Friel et al. observed an intact bone-tendon junction at two weeks after acute rotator cuff repair with abundant scar formation on macroscopic evaluation (22). At eight weeks post-repair, parallel orientation of collagen fibrils, decreased cellularity, and an absence of vascularisation was noted. No difference was noted between two and eight weeks after repair with regard to the semiquantitative evaluation. Concerning the control groups with Ethibond only repair, all failures were at the suture-tendon repair site.

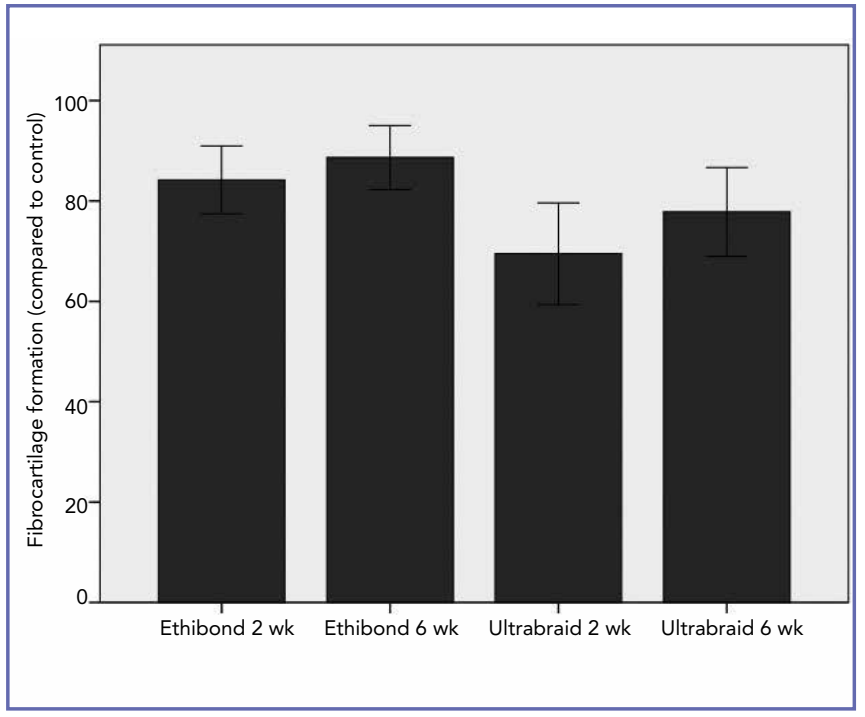

Figure 6. Semiquantitative histologic scoring of all the groups.

Su et al. investigated the effect of suture absorbability on acute rotator cuff healing and found that nonabsorbable sutures (PDS) have better biomechanical and histologic properties than partially absorbable (Orthocord) and nonabsorbable sutures (Ethibond) (7). This finding was attributed to the stress-shielding effect of nonabsorbable sutures; however, no data on repair using different suture materials in-vivo and protein expressions was provided (7). Contrarily, Sun et al. found no obvious inflammation at three weeks and nine weeks after repair with nonabsorbable Ethibond sutures and observed good tendon-bone healing (14). Placing knots on the bone, even with the use of Ethibond, results in a comparable improvement in histologic scores, regardless of the suture material used, which confirms the findings of the current study.

All studied structural proteins and enzymes that have an impact on rotator cuff healing have been shown to be elevated in each repair group. IGF-1, TGF-beta 1, MMP-2, and COX-2 levels are elevated at two weeks and six weeks after repair. This could be explained by the proliferation and remodelling phase, similar to the acute healing process, which can last up to eight weeks (23). Galatz et al. found that collagen I and III mRNA levels were high for 56 days in an acute rotator cuff tear model (24). Similarly, Würgler-Hauri et al. have demonstrated increased TGF-beta 1 synthesis at healing entheses at eight weeks and 16 weeks after a tenotomy in a rat model (25). Increased TGF-beta 1 synthesis at an earlier time point (two weeks) in suture repair groups refers to a fibrotic reaction against suture materials, which 


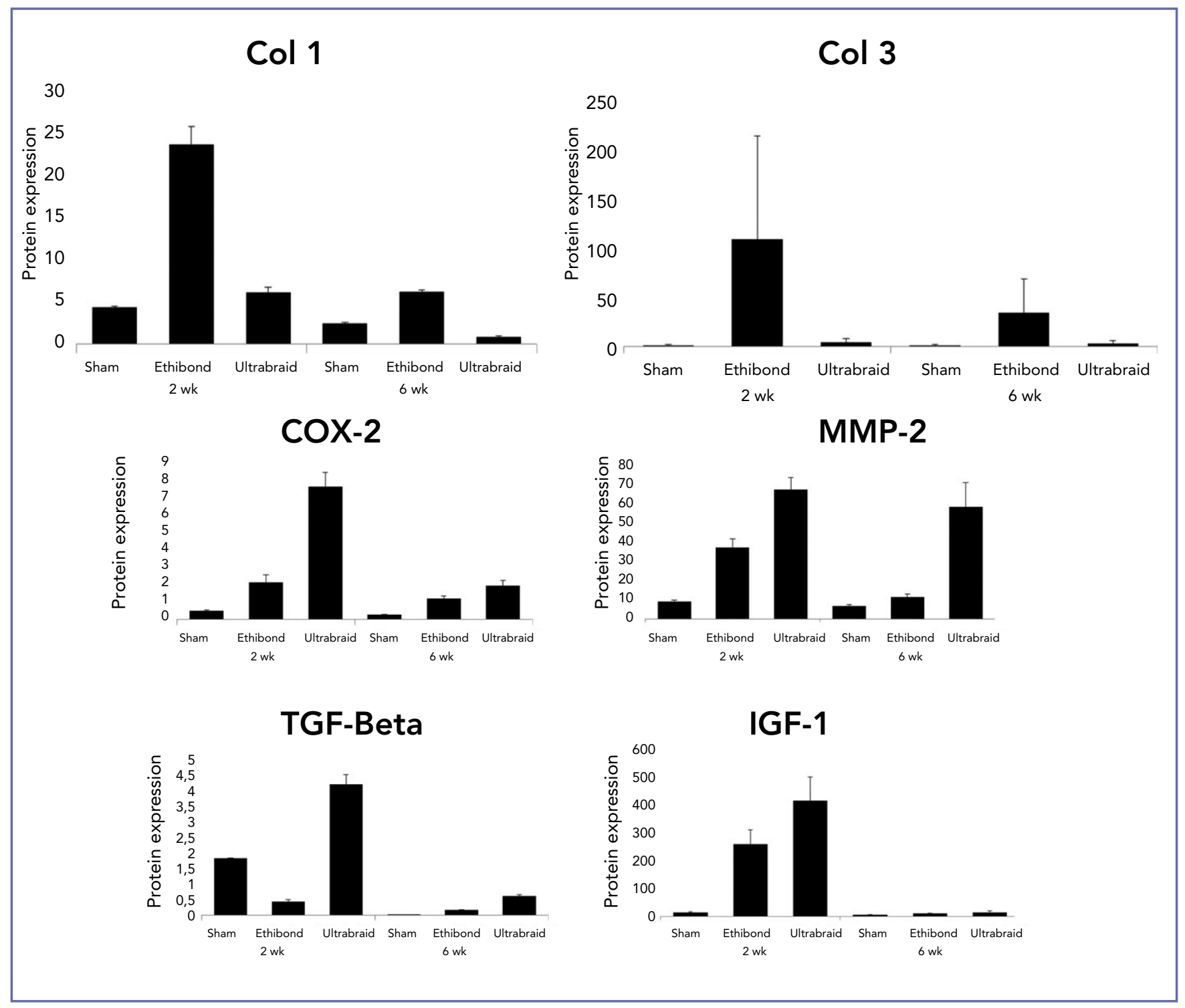

Figure 7. Protein fold expression of the different markers in relation to rotator cuff healing (compared to the controls). The western blot demonstrated a significant temporal decrease in protein expression following repair with both suture types. There is a significant difference between the two different suture groups at two weeks and six weeks after repair.

EB: Ethibond; UB: Ultrabraid.

is supported by the presence of the scar tissue detected in the suture-tendon interfaces of this study's suture repair groups. Although IGF-1 is an anabolic factor, it is elevated in the early phase after repair. Increased expression of IGF-1, along with MMP-2 and COX-2, implies its regulatory function in inflammation, which requires further investigation in prospective studies. Markers related to impaired rotator cuff healing, namely MMP-2 and COX-2, were obviously elevated in the Ultrabraid groups. This finding could explain the tendency for increased fibrocartilage formation in favour of the Ethibond suture at each time point. Finally, one clinical study found similar outcomes after transosseous repair with both nonabsorbable Ethibond and absorbable PDS sutures (26), confirming similar histologic findings after repair with different suture materials, as in the current study. Although recent literature has focused on the development of coatings for sutures with various growth factors to enhance tendon-to-bone healing (27), prospective clinical 
and basic science studies investigating chronic rotator cuff healing could further confirm the findings of the current study by coating suture materials with COX-2 (ibuprofen) or MMP-2 inhibitors (doxycycline) $(28,29)$. This study could further direct new therapeutic targets as mentioned by Montiel Terrón et al. (30).

This study has several strengths. In addition to the effects of the suture materials, it provides insight into protein expressions in a chronic rotator cuff tear model. Temporal changes in collagen production, fibrotic (TGF-beta 1), anabolic (IGF-1), inflammatory, and catabolic markers (COX-2, MMP-2) were observed. Two different time points allowed the observation of time-dependent changes. Aged subjects were used to increase clinical adaptation. However, further studies are required, as there is insufficient evidence regarding the histopathological analysis of the rotator cuff healing (31).

This is a preliminary study. The limitations of this study include the use of an animal model; thus, any conclusions cannot be assumed to apply to humans or have any clinical significance. In addition, only two different time points were used, namely two weeks and six weeks, and rotator cuff tendon healing takes much longer in a clinical setting. Further, the activity of fibroblasts and adipocytes, which take part in rotator cuff muscle fatty infiltration, were not evaluated (32), and only one type of suture repair stitch, specifically the Mason-Allen, was tested; hence, this data may not apply to other stitch configurations or to repairs using suture anchors.

\section{REFERENCES}

1. Galatz LM, Ball CM, Teefey SA, Middleton WD, Yamaguchi $\mathrm{K}$. The outcome and repair integrity of completely arthroscopically repaired large and massive rotator cuff tears. JBJS 2004;86(2):219-24.

2. Robertson CM, Chen CT, Shindle MK, Cordasco FA, Rodeo SA, Warren RF. Failed healing of rotator cuff repair correlates with altered collagenase and gelatinase in supraspinatus and subscapularis tendons. Am J Sports Med 2012;40(9):1993-2001.

3. Angeline ME, Dines JS. Factors affecting the outcome of rotator cuff surgery. In: Nicholson GP (eds). Orthopaedic knowledge update: Shoulder and elbow 4. American Academy of Orthopaedic Surgeons, 2013:pp. 217-28.

4. Voleti PB, Buckley MR, Soslowsky LJ. Tendon healing: repair and regeneration. Annu Rev Biomed Eng 2012;14:47-71.

5. Clement N, Hallett A, MacDonald D, Howie C, McBirnie J. Does diabetes affect outcome after arthroscopic repair of the rotator cuff? J Bone Joint Surg Br 2010;92(8):1112-7.

6. Galatz L, Silva M, Rothermich S, Zaegel M, Havlioglu N, Thomopoulos S. Nicotine delays tendon-to-bone healing in a rat shoulder model. JBJS 2006;88(9):2027-34.

7. Su W, Qi W, Li X, Zhao S, Jiang J, Zhao J. Effect of suture absorbability on rotator cuff healing in a rabbit rotator cuff repair model. Am J Sports Med 2018;46(11):2743-54.

\section{CONCLUSIONS}

In this chronic rotator cuff model, repair with both Ultrabraid and Ethibond sutures resulted in a similar histologic response, but there were different levels of protein expression at two weeks and six weeks after repair according to the western blot analysis.

The study was conducted according to the journal's standards (33).

\section{ACKNOWLEDGEMENTS}

This article has been extracted from a thesis submitted to Marmara University Medical Faculty in fulfillment of the requirement for the degree of specialty in orthopaedic surgery.

\section{SOURCE OF FUNDING}

It was supported by Marmara University Scientific Research Commission (SAG-C-TUP-131216-0527) and Turkish Orthopaedic Research Council (date and number: 01.03.2017/4).

\section{CONFLICT OF INTERESTS}

The authors declare that they have no conflict of interests.

8. Wüst DM, Meyer DC, Favre P, Gerber C. Mechanical and handling properties of braided polyblend polyethylene sutures in comparison to braided polyester and monofilament polydioxanone sutures. Arthroscopy 2006;22(11):1146-53.

9. Haslam SG, Barber FA. Anchors and Sutures. In: Milano G, Grasso A (eds). Shoulder Arthroscopy. London: Springer, 2014:pp 135-48.

10. Barber FA, Herbert MA, Coons DA, Boothby $\mathrm{MH}$. Sutures and suture anchors-update 2006. Arthroscopy 2006;22(10):1063-9.e2.

11. Lock AM, Gao R, Naot D, Coleman B, Cornish J, Musson DS. Induction of immune gene expression and inflammatory mediator release by commonly used surgical suture materials: an experimental in vitro study. Patient Saf Surg 2017;11(1):1-8.

12. Ergün S, Alakbarov A, Yılmaz A, Karademir B, Akgün U. The Effect of Different Suture Materials on Achilles Tendon Metabolism: A Preliminary in vivo Study of mRNA levels in Rabbits. MLTJ 2019;9(4).

13. Owens BD, Algeri J, Liang V, DeFroda S. Rotator cuff tendon tissue cut-through comparison between 2 high-tensile strength sutures. J Shoulder Elbow Surg 2019. 
14. Sun Y, Kwak J-M, Kholinne E, Tan J, Koh K-H, Jeon I-H. Nonabsorbable Suture Knot on the Tendon Affects Rotator Cuff Healing: A Comparative Study of the Knots on Tendon and Bone in a Rat Model of Rotator Cuff Tear. Am J Sports Med 2019;47(12):2809-15.

15. Otarodifard K, Wong J, Preston CF, Tibone JE, Lee TQ. Relative fixation strength of rabbit subscapularis repair is comparable to human supraspinatus repair at time 0. Clin Orthop Relat Res 2014;472(8):2440-7.

16. Gupta R, Lee TQ. Contributions of the different rabbit models to our understanding of rotator cuff pathology. J Shoulder Elbow Surg 2007;16(5):S149-S57.

17. Rowshan K, Hadley S, Pham K, Caiozzo V, Lee TQ, Gupta R. Development of fatty atrophy after neurologic and rotator cuff injuries in an animal model of rotator cuff pathology. JBJS 2010;92(13):2270-8.

18. Oh JH, Chung SW, Kim SH, Chung JY, Kim JY. 2013 Neer Award: Effect of the adipose-derived stem cell for the improvement of fatty degeneration and rotator cuff healing in rabbit model. J Shoulder Elbow Surg 2014;23(4):445-55.

19. Zhang S, Li H, Tao H, et al. Delayed early passive motion is harmless to shoulder rotator cuff healing in a rabbit model. Am J Sports Med 2013;41(8):1885-92.

20. Chung SW, Song BW, Kim YH, Park KU, Oh JH. Effect of platelet-rich plasma and porcine dermal collagen graft augmentation for rotator cuff healing in a rabbit model. Am J Sports Med 2013;41(12):2909-18.

21. Fox AJ, Schär MO, Wanivenhaus F, et al. Fluoroquinolones impair tendon healing in a rat rotator cuff repair model: a preliminary study. Am J Sports Med 2014;42(12):2851-9.

22. Friel NA, Wang VM, Slabaugh MA, Wang F, Chubinskaya S, Cole BJ. Rotator cuff healing after continuous subacromial bupivacaine infusion: an in vivo rabbit study. J Shoulder Elbow Surg 2013;22(4):489-99.

23. Zgonis MH, Andarawis NA, Soslowsky LJ. Mechanics and healing of rotator cuff injury. In: Abrams JS, Bell
RH (eds). Arthroscopic Rotator Cuff Surgery. Springer, 2008:pp. 332-47.

24. Galatz LM, Sandell LJ, Rothermich SY, Das R, Mastny A, Havlioglu N, et al. Characteristics of the rat supraspinatus tendon during tendon-to-bone healing after acute injury. J Orthop Res 2006;24(3):541-50.

25. Würgler-Hauri CC, Dourte LM, Baradet TC, Williams GR, Soslowsky LJ. Temporal expression of 8 growth factors in tendon-to-bone healing in a rat supraspinatus model. J Shoulder Elbow Surg 2007;16(5):S198-S203.

26. Boehm T, Werner A, Radtke S, Mueller T, Kirschner S, Gohlke F. The effect of suture materials and techniques on the outcome of repair of the rotator cuff: a prospective, randomised study. J Bone Joint Surg Br 2005;87(6):819-23.

27. Beitzel K, Voss A, McCarthy M-B, et al. Coated Sutures. Sports Med Arthrosc Rev 2015;23(3):e25-e30.

28. Bedi A, Kovacevic D, Hettrich C, et al. The effect of matrix metalloproteinase inhibition on tendon-to-bone healing in a rotator cuff repair model. J Shoulder Elbow Surg 2010;19(3):384-91.

29. Oak NR, Gumucio JP, Flood MD, et al. Inhibition of 5-LOX, COX-1, and COX-2 increases tendon healing and reduces muscle fibrosis and lipid accumulation after rotator cuff repair. Am J Sports Med 2014;42(12):2860-8.

30. Montiel Terrón V, Muiños-López E, Granero-Moltó F, et al. Muscular injuries after tendon rupture in the rotator cuff of animal models. Systematic review. MLTJ 2018;8(2).

31. Cipollaro L, Sahemey R, Oliva F, Maffulli N. Immunohistochemical features of rotator cuff tendinopathy. Br Med Bull 2019;130(1):105-23.

32. Liu X, Ning AY, Chang NC, et al. Investigating the cellular origin of rotator cuff muscle fatty infiltration and fibrosis after injury. MLTJ 2016;6(1):6.

33. Padulo J, Oliva F, Frizziero A, Maffulli N. Muscles, Ligaments and Tendons Journal - Basic principles and recommendations in clinical and field Science Research: 2018 update. MLTJ 2018;8(3):305-307. 